\title{
ON A FUNDAMENTAL EVALUATION OF A UAV EQUIPPED WITH A MULTICHANNEL LASER SCANNER
}

\author{
K.Nakano $^{\text {a,b, } *, \text { H. Suzuki }^{a}, \text { K. Omori }}{ }^{\text {a }}$, K. Hayakawa ${ }^{\text {c }}$, M. Kurodai ${ }^{\text {c }}$ \\ Geospatial Information Laboratory, AERO ASAHI CORPORATION, \\ 3-14-4, Minamidai, Kawagoe, Saitama, 350-1165, Japan - kazuya-nakano@aeroasahi.co.jp \\ ${ }^{\mathrm{b}}$ Division of Architectural, Civil and Environmental Engineering, Tokyo Denki University, \\ Ishizaka, Hatoyama, Saitama, 350-0394, Japan \\ ${ }^{\mathrm{c}}$ Technical Research Institute, HAZAMA ANDO CORPORATION, \\ 515-1, Karima, Tsukuba, Ibaraki, 305-0822, Japan
}

Commission II, ICWG I/II

KEY WORDS: UAV, Multichannel laser scanner, Accuracy evaluation, Laser-acquired point cloud, Photogrammetry

\begin{abstract}
:
Unmanned aerial vehicles (UAVs), which have been widely used in various fields such as archaeology, agriculture, mining, and construction, can acquire high-resolution images at the millimetre scale. It is possible to obtain realistic 3D models using highoverlap images and 3D reconstruction software based on computer vision technologies such as Structure from Motion and Multiview Stereo. However, it remains difficult to obtain key points from surfaces with limited texture such as new asphalt or concrete, or from areas like forests that may be concealed by vegetation. A promising method for conducting aerial surveys is through the use of UAVs equipped with laser scanners. We conducted a fundamental performance evaluation of the Velodyne VLP-16 multi-channel laser scanner equipped to a DJI Matrice 600 Pro UAV at a construction site. Here, we present our findings with respect to both the geometric and radiometric aspects of the acquired data.
\end{abstract}

\section{INTRODUCTION}

Three-dimensional reconstruction of SfM (Structure from Motion)/ MVS (Multi-view Stereo) using high-resolution and high overlap images via Unmanned Aerial Vehicle (UAV) has become a common measurement method used for threedimensional surveying. i-Construction, which aims to improve the efficiency of construction work proposed by the Ministry of Land, Infrastructure and Transport in Japan provides one use for UAV surveys. However, it can be difficult to recover accurate ground surface information in forested areas, a problem that is shared with other photogrammetry methods; UAVs are expected to use laser scanners similar to the ones employed in airborne laser surveys.

Equipping UAVs with laser scanners has been under consideration for some time. For example, Nagai et al. produced a mapping system that integrated an inexpensive GPS, inertial measurement unit (IMU), digital camera, and SICK $18 \mathrm{~Hz}$ laser scanner using an unmanned helicopter in 2003. In more recent studies, the use of lightweight laser scanners from Hokuyo Electric and Velodyne has been facilitated by the increased accessibility of multi-copters. Surveys have also been conducted using dedicated Riegl VUX-1 UAVs, and amphibious landforms equipped with green laser profilers.

The performance of both the direct georeferencing device and the laser distance measuring device greatly affects the accuracy of UAVs equipped with laser scanners. It is commonly inferred that high-accuracy imaging is incompatible with the operation of lightweight and inexpensive UAVs due to the required increases in equipment cost and weight. However, when taking into consideration general UAV performance, flight time, payload, and reliability, it may be reasonable to choose a lightweight and relatively inexpensive laser scanner for threedimensional surveys. There are several laser scanner units for UAVs on the market; we adopted products by LiDAR USA that combined GNSS / IMU, and a Velodyne VLP-16 laser scanner, which was primarily built as a low-cost, collision avoidance laser scanner for the automotive industry.

In this study, we evaluate the fundamental performance of UAVs equipped with laser scanners. Data was acquired from a construction site, and accuracy was assessed, including by comparison with three-dimensional reconstructions by images. We also analyzed specific characteristics of the utilized multichannel laser scanner.

\section{EQUIPMENT FOR EVALUATION}

For the fundamental performance evaluation, we used a DJI Matrice 600 Pro UAV, and LiDAR USA laser scanner units. The appearance from the rear of the UAV equipped with the laser scanner is shown in Figure 1, and the specifications of the UAV are shown in Table 1.

The specifications of the laser scanner unit are shown in Table 2. Velodyne VLP-16 is a multichannel laser scanner that irradiates 16 laser beams shifted $2^{\circ}$ in the front and rear with respect to the flight direction, and scans $360^{\circ}$ in each direction perpendicular to the flight direction to obtain data. It is therefore expected that the ground acquisition rate can be improved by penetrating dense vegetation by 16 laser irradiations with different incident angles.

\footnotetext{
* Corresponding author
} 


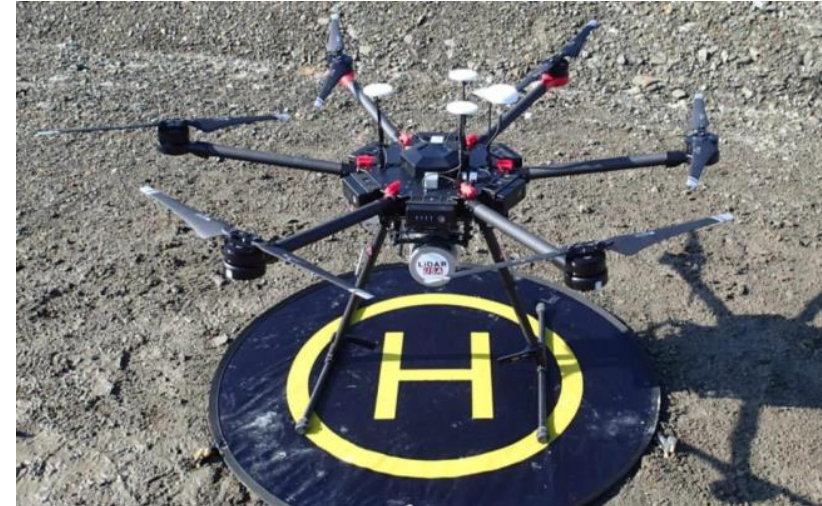

Figure 1. DJI Matrice 600 Pro UAV

\begin{tabular}{|l|l|}
\hline Item & Specifications \\
\hline \hline Rotors & 6 \\
\hline Flight controller & DJI A3 Pro \\
\hline Flight log & View only \\
\hline Weight & ca. 13 kg (with battery, scanner) \\
\hline Flight time & ca. 20 minutes \\
\hline Autonomous flight & Leading waypoints \\
\hline
\end{tabular}

Table 1. UAV specifications

\begin{tabular}{|c|c|}
\hline Item & Specifications \\
\hline "GNSS/IMU & בApplanix APX-15UAV \\
\hline Positioning accuracy & $0.02 \sim 0.05 \mathrm{~m}$ \\
\hline Roll, Pitch & 0.025 deg. \\
\hline Heading & $0.080 \mathrm{deg}$. \\
\hline Laser scanner & Velodyne VLP-16 \\
\hline Pulse rate & $300,000 \mathrm{~Hz}$ \\
\hline Max distance & $100 \mathrm{~m}$ \\
\hline Distance accuracy & $\pm 3 \mathrm{~cm}$ \\
\hline FOV & $\begin{array}{l}30 \text { deg. (Flight direction) } \\
360 \text { deg. (Perpendicular) }\end{array}$ \\
\hline Channel & 16 \\
\hline Total Weight & ca. $2 \mathrm{~kg}$ \\
\hline
\end{tabular}

Table 2. Laser scanner specifications

To evaluate the point clouds rendered by the laser scanner, images were obtained using an Alpha a6000 digital camera manufactured by Sony Corporation for three-dimensional reconstruction; camera specifications are shown in Table 3.

\begin{tabular}{|l|l|}
\hline Item & Specifications \\
\hline \hline Focal length & $16 \mathrm{~mm}$ \\
\hline Sensor size & APS-C size $(23.4 \times 15.6 \mathrm{~mm})$ \\
\hline Image size & $6000 \times 4000$ \\
\hline Pixel size & $3.9 \mu \mathrm{m}$ \\
\hline
\end{tabular}

Table 3. Digital camera specifications

\section{DATA ACQUISITION}

Data for the fundamental performance evaluation was obtained by laser scanner and digital camera for 3D reconstruction, and coordinate observation of the test target using GNSS and Total Station was performed.

\subsection{Work area and test target observation}

Data for evaluation was acquired over a work area of approximately $200 \mathrm{~m} \times 100 \mathrm{~m}$ at a site under construction on residential land. The work area includes a flat area and a slope face, with a maximum height difference of $\sim 35 \mathrm{~m}$. Trees can be seen around the slope face in the upper part of Figure 2; exist tree height was $\sim 20 \mathrm{~m}$. The coordinates of two reference points were acquired by GNSS static observation, and the coordinates for 62 points on the test targets were observed by radiation method using Total Station.

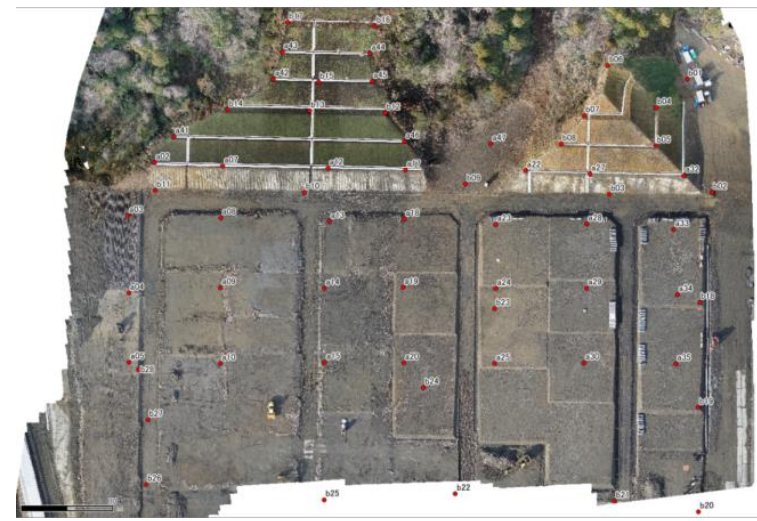

Figure 2. Work area construction site; numbers beginning with $\mathrm{a}$ or $\mathrm{b}$ indicate test targets.

\subsection{Laser data acquisition}

Measurements by UAV equipped with a laser scanner were performed with several settings. The point cloud data used for accuracy evaluation was generated over 3 courses at a flight height from a flat area of $40 \mathrm{~m}$, and a flight speed of $3 \mathrm{~m} / \mathrm{s}$. Figure 3 shows a tint map in which the point cloud data are assigned to blue to red over the range of $0 \mathrm{~m}$ to $55 \mathrm{~m}$ in relative height. Point cloud data was acquired up to $\sim 55 \mathrm{~m}$, even at a flight height of $40 \mathrm{~m}$ used to irradiate a laser beam of $360^{\circ}$ directed perpendicular to the flight direction.

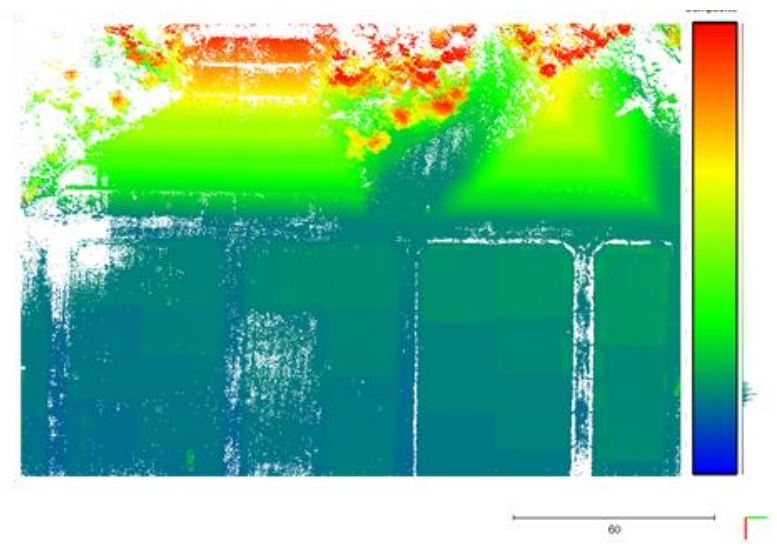

Figure 3. Tint map of laser data

\subsection{Image acquisition for reference data}

Three-dimensional reconstruction of the work area by digital camera images was performed using PhotoScan Professional for comparative evaluation of laser scanned images after the work procedure for i-Construction operated by Hazama Ando Corporation. This work procedure is designed to satisfy 
Japanese regulations for public surveying and construction surveys using UAVs. The image acquisition specifications were: $1 \mathrm{~cm}$ for the ground sample distance, $90 \%$ overlap, and $60 \%$ side lap. As the photographing reference plane satisfies the required $1 \mathrm{~cm}$ of the ground sample distance, it was divided into several reference planes at the slope face. The image acquisition area was enlarged to cover the measurement area of the laser scanner. It was used to test 16 target points as the ground control point that satisfies the regulation for the arrangement of three-dimensional reconstructions.

\section{FUNDAMENTAL PERFORMANCE EVALUATION}

For the performance evaluation in this study, we quantitatively compared the coordinates of test targets with the results of a three-dimensional reconstruction achieved by multichannel laser scanner, and qualitatively evaluated a cross-section of the vegetated area.

The processing of laser scanner data was carried out using the ScanLook PC dedicated application of LiDAR USA. In the procedure, after analyzing the flight trajectory of GNSS / IMU by POSPac, and distance data from the Velodyne VLP - 16, integrated analyses were performed. The ScanLook PC has a function that allows for adjustment points, but there are situations when it is not always possible to set adjustment points, such as in areas affected by natural disaster. Therefore, two analytical scenarios were evaluated on the condition of the presence or absence of adjustment points.

\subsection{Evaluation using coordinates of test targets}

Accuracy of point cloud data acquired by laser scanner was quantitatively evaluated using test targets. The test targets used two kinds of planar shapes, one was a $20 \mathrm{~cm}$ square and the other was A3 paper-sized. It is difficult to recognize the shape of the test target in the point cloud of the laser scanner discretely acquired by scan mechanism. Therefore, the accuracy was evaluated by using the height value interpolated from the coordinates of the neighboring point cloud on the planimetric coordinates of the test targets. The most probable value of the height was calculated by the Inverse Distance Weighted method using point cloud data included at a radius of $10 \mathrm{~cm}$ around the planimetric coordinates of the test target.

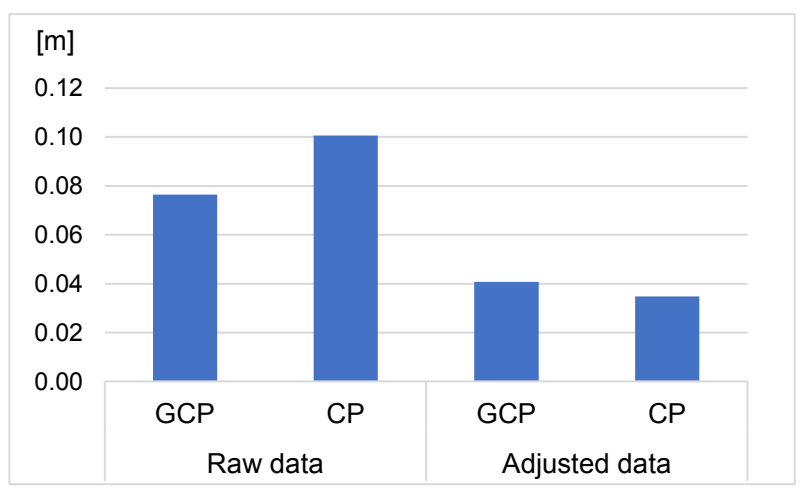

Figure 4. RMSE for ground points

Figure 4 shows the root mean square error (RMSE) calculated from the most probable value of ground control points (GCP) and check points (CP). A total number of 48 points were evaluated; $t 9$ ground control points, and 39 check points were used. The RMSE, presented in Figure 4, is approximately \pm 10 $\mathrm{cm}$ before adjustment and is reduced to approximately $\pm 4 \mathrm{~cm}$ by adjustment. Figure 5 shows the results of summarizing the average of the difference values of the heights used to calculate the RMSE in order to check for systematic error in the point cloud data before adjustment.

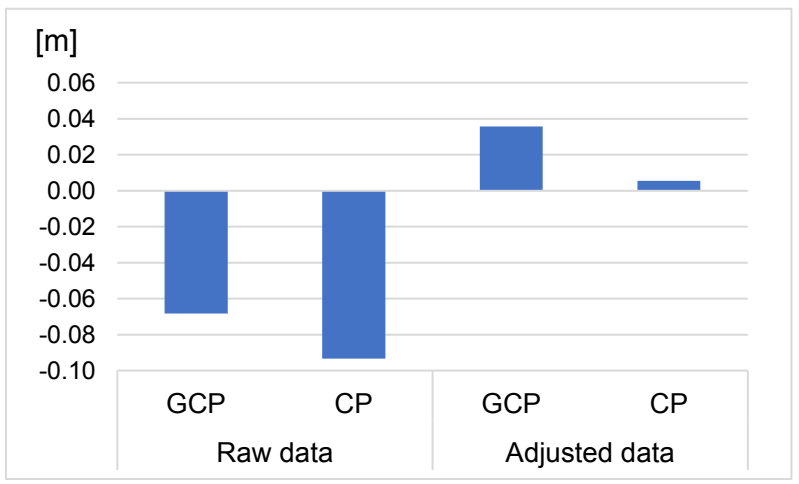

Figure 5. Results of summarizing the average

From Figure 5, it is inferred that some error was included in direct georeferencing because the average before adjustment is uniformly negative.

\subsection{Evaluation using 3D reconstruction results}

The RMSE of height was $\pm 0.013 \mathrm{~m}$ which was obtained for 63 test targets by the same evaluation method in the previous section. Three-dimensional point clouds were generated by $3 \mathrm{D}$ reconstruction created through an applicable procedure in ICT Earthwork. The results of comparing the three-dimensional point cloud data collected by UAV equipped with a laser scanner after adjustment, and the three-dimensional point cloud data generated by $3 \mathrm{D}$ reconstruction are shown in Figure 6. Our results indicate that the greatest height differences were less than $0.10 \mathrm{~m}$, and provide insight into the effectiveness of the Velodyne VLP-16 laser scanner.

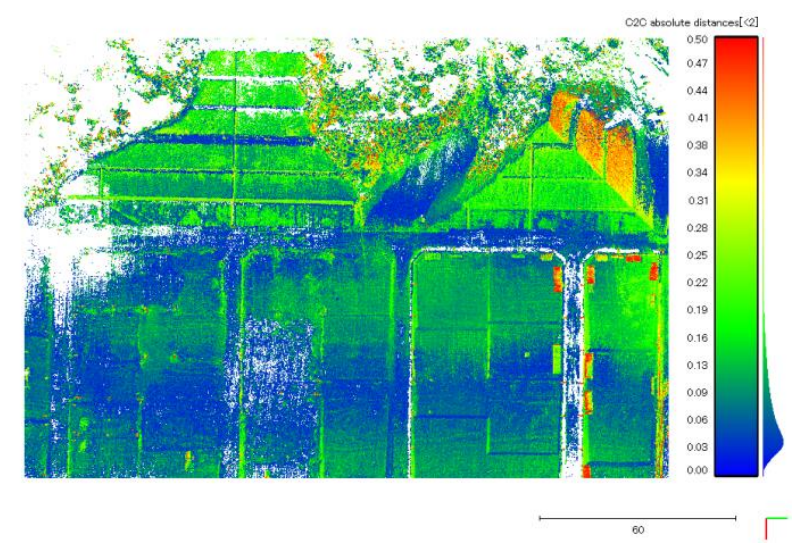

Figure 6. Results of comparing the point cloud data

\subsection{Qualitative evaluation using cross section of tree area}

In order to confirm the acquisition of the ground surface over the forested area which is the characteristic of the laser scanner, we evaluated a cross section of the area using point cloud data from $3 \mathrm{D}$ reconstruction and the laser scanner (Figure. 7). As shown in Figure 7, the point cloud generated by 3D reconstruction acquires the tree surface, whereas the point cloud generated by the laser scanner passes through the leaves and 
acquires the ground surface. The point indicated by the brokenline circle is the flight trajectory.

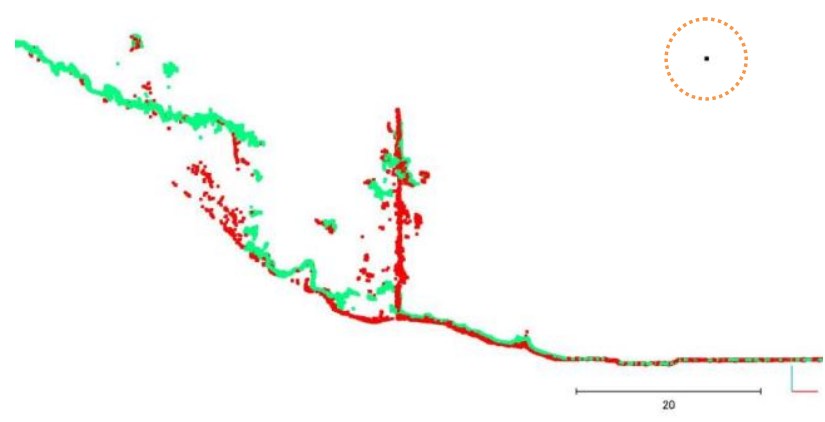

Figure 7. Cross section of the forested area. Green indicates the point cloud of the laser scanner, and red indicates the point cloud of photographic $3 \mathrm{D}$ reconstruction.

\section{DISCUSSION}

\subsection{Missing parts of laser scanner-generated point cloud}

The missing parts of the point cloud data are shown in the tint map of the laser scanner (Figure 3).

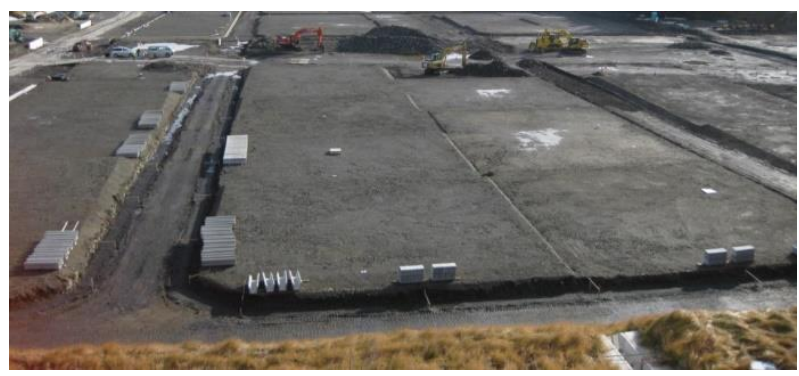

Figure 8. Work area with visible puddles

Precipitation at the time of data collected contributed to gaps in laser scanner-generated point clouds. Rainfall and snowfall occurred the day before the measurements were taken, and there were several puddles, as shown in Figure 8; on the day of measurement, the soil was in a muddy condition. It is therefore inferred that gaps in the data are due to the reflection not being obtainable with Class 1 laser scanner used because of high soil moisture.

\subsection{Characteristics of the multichannel laser}

Quantitative evaluation revealed that the height accuracy of the point cloud of the adjusted multichannel laser was $\pm 4 \mathrm{~cm}$. However, there were some deviations to most probable value revealed by the Inverse Distance Weighted method. This is possibly due to the fact that the multichannel laser scanner irradiates 16 laser beams. Therefore, characteristics of multichannel laser scanner were examined.

\subsubsection{Evaluation using the point cloud footprint}

The irradiation points of the laser beam of the multi-channel laser scanner are almost identical; the FOVs at the front and rear are $30^{\circ}$, and the left and right FOVs are $360^{\circ}$. It is estimated that the footprint shape of the point cloud spreads radially. Figure 9 shows the footprint acquired from an altitude of $20 \mathrm{~m}$ over the flat area.

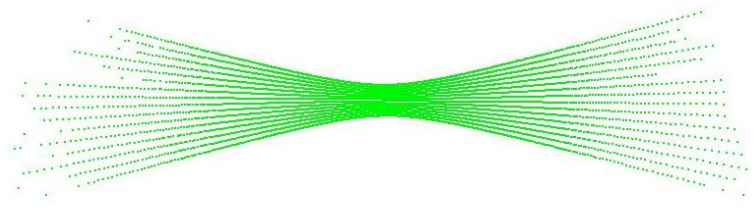

Figure 9. Footprint over the flat area

It is understood from Figure 9 that the laser beam spreads in a radial direction in a drum shape due to the effect of the laser beam's inclined irradiation. A characteristic of the multichannel laser scanner is that the vertical laser beam uniformly scans the direction perpendicular to the flight direction like the airborne laser scanner, and the other inclined laser beams scan nonuniformly according to distance and angle. However, when the UAV flying at constant speed and equal altitude, the conditions of the respective channels are the same and it is considered that the uniformity of each channel is kept.

\subsubsection{Evaluation using the number of points}

The number of points acquired for each channel was analyzed to evaluate the point cloud generated by the multichannel laser scanner. The results with the counted number of acquired points on the vertical axis and the channel on the horizontal axis are shown in Figure 10. That there is a large difference between the number of points between odd- and even-numbered channels is easily recognized. The channel number varies in the direction of tilt by odd-numbered and even-numbered channels. The odd channels are set from vertical view to the backward view, and the even numbers are set from the forward view to vertical view relative to the flight direction. There is a tendency for the number of points in the forward view of even channels to be reduced, while the channels that captured the backward view or the vertical view could acquire large numbers of points. Channel 11 has the largest number of points as well as a large tilt angle of $11^{\circ}$. It is inferred that this is due to variations in the sensor because the trend is different from that of the other channels.

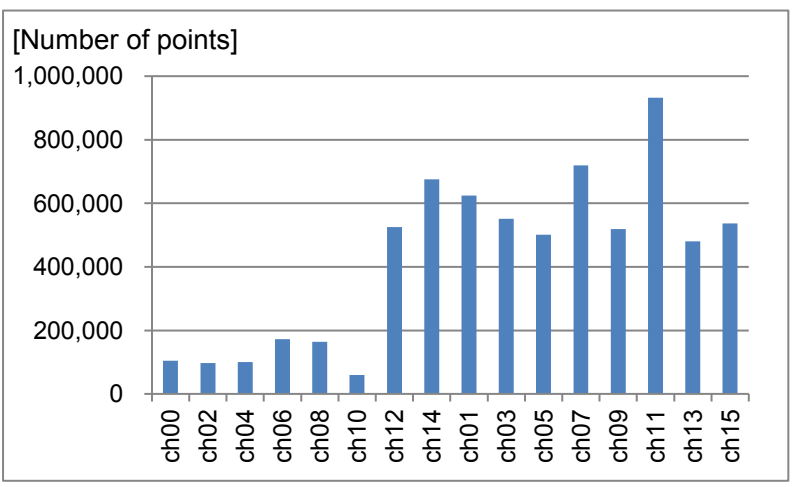

Figure 10. Counted number of acquired points by laser channel with even- and odd-numbered channels appearing the left and right of the plot, respectively

\subsubsection{Evaluation using reflection intensity}

As the number of acquisition points greatly differs depending on the channel, further evaluation was conducted using the reflection intensity of the point cloud. It is assumed that the reflection intensity decreases as the tilt angle increases because the angle of incidence of the laser beam is different for each channel. 
The average values of the reflection intensity and the results of calculating the deviation of the point cloud data for each channel are shown in Figure 11. It can be determined from these results that the average values of the even channels indicate high reflection intensity. Considering that the number of points to acquired was small, it is inferred that this is due to the acquisition of a point where reflection was strongly obtained. Such inference is also supported by the fact that the deviation of the corresponding channel shows a small value. However, channels with a large number of acquired points exhibit a tendency for the acquired reflection intensities to be small, with large deviations. Combining the reflection intensities with these deviations is presumed to reduce the interpretations of the reflection intensity alone.

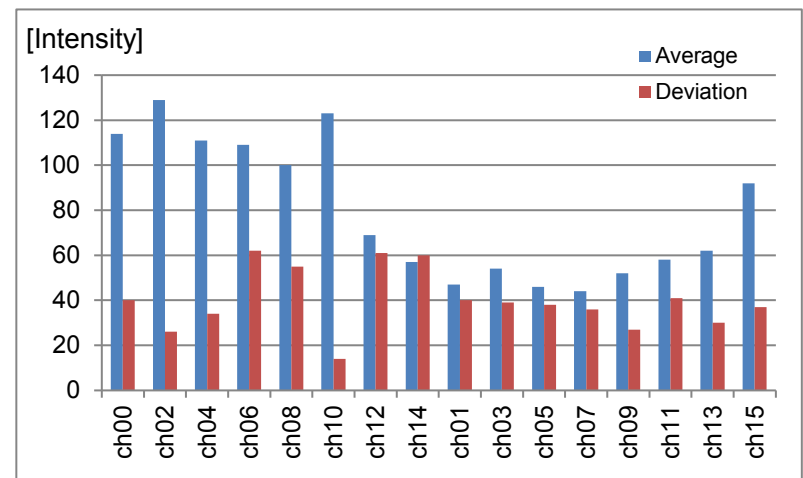

Figure 11. Analysed results of reflection intensity

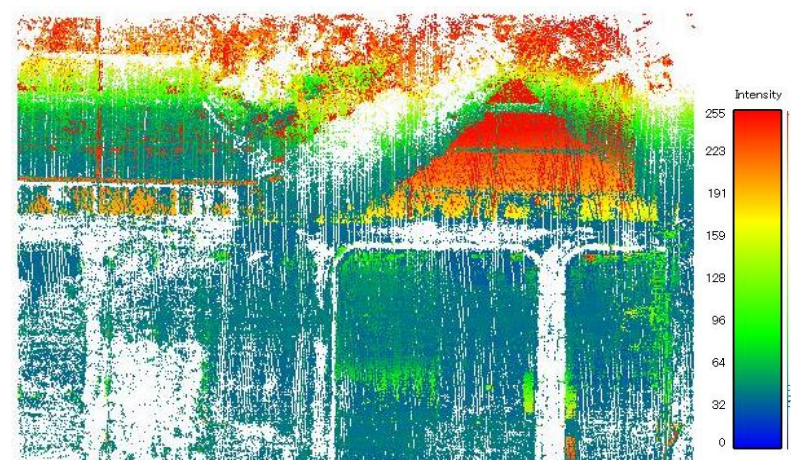

Figure 12. Reflection intensity image of channel 8

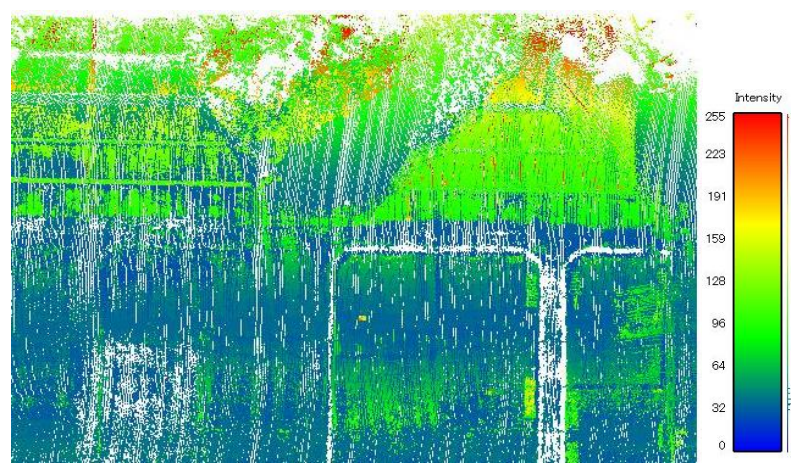

Figure 13. Reflection intensity image of channel 11

Furthermore, reflection intensity was evaluated for some specific channels in this study. The reflection intensity images of channels 8 and 11 are shown in Figure 12 and Figure 13, respectively; channel 8 reveals a high reflection intensity. Our results suggest that concrete and vegetation yield a high reflectivity, which emerge in the strong tendency of reflection observed in each channel.

Comparing Figure 12 and Figure 13 suggests that intensity value generally increases with altitude. In order to better understand the relationship between the reflection intensity and altitude, we analyzed reflection intensity relative to the scanning angle; Figure 14 presents a histogram of this relationship between. The scanning angle of $-90^{\circ}$ is the nadir direction and $0^{\circ}$ is the horizontal direction in Figure 14. Although there is a difference in the reflection intensity between channels 8 and 11, the reflection intensity tends to increase as the scanning angle increases. From the results obtained in these evaluations, we suggest that the reflection intensity of the Velodyne VLP - 16 multichannel laser scanner is positively correlated with the scanning angle.

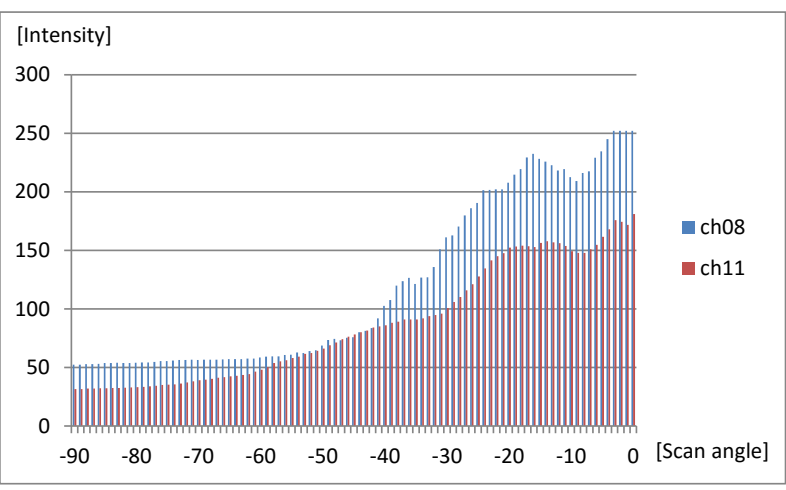

Figure 14. Reflection intensity and scan angles

\section{CONCLUSIONS}

A Fundamental performance evaluation of UAV equipped with a Velodyne VLP - 16 multichannel laser scanner was conducted. Quantitative evaluations using test targets for comparison with three-dimensional photographic reconstructions, and qualitative evaluations using a cross-section of the forested area were made. Our results reveal that the root mean square error is within \pm 10 $\mathrm{cm}$, even for point clouds without adjustment points. The error was further reduced to approximately $\pm 4 \mathrm{~cm}$ by the adjustment using ground control points. The effectiveness of the laser scanner was also demonstrated by comparison with photographic 3D reconstruction, and a cross section of the forested zone. The characteristics of the multichannel laser scanner were further evaluated relative to the drum-shaped footprint, and both the number of points and the reflection intensity variation among channels. It was expected that the information acquired would increase by using the multichannel laser scanner. However, to interpret such information, variations in point cloud resolution must be considered from the viewpoint of the geometric and radiometric characteristics of multichannel laser scanner. Further studies, with increased data, more favorable field conditions, and additional sensor calibration are needed to develop a more complete understanding of the fundamental performance capabilities of UAV-mounted multichannel laser scanners.

\section{ACKNOWLEDGEMENTS}

We have received a great deal of technical cooperation for our measurements and analyses of LiDAR USA and Velodyne VLP-16 multi-channel laser scanner-equipped DJI Matrice 600 Pro UAV from Mr. T. Iwabu of Nikon Trimble Co., Ltd. We would like to express our thanks to the cooperation. 


\section{REFERENCES}

G. Mandlburger, M. Pfennigbauer, M. Wieser, U. Riegl, N. Pfeifer, 2016. Evaluation of a Novel UAV-Borne TopoBathymetric Laser Profiler, The International Archives of the Photogrammetry, Remote Sensing and Spatial Information Sciences, XLI-B1, pp.933-939.

K.-D. Kuhnert and L. Kuhnert, 2013. Light-Weight Sensor Package for Precision 3D Measurement with Micro UAVs e.g. Power-Line Monitoring, The International Archives of the Photogrammetry, Remote Sensing and Spatial Information Sciences, XL-1/W2, pp.235-240.

M. Nagai, R. Shibasaki, H. Kumagai, S. Mizukami, D. Manandhar, H. Zhao. 2004. Construction of Digital Surface Model by Multisensor Integration from an Unmanned Helicopter. ISPRS Archives - Volume XXXVI-5/W1, WG V/6, Processing and Visualization using High-Resolution Images

T. Gee, J. James, W. V. D. Mark, P. Delmas, G. Gimel'farb, 2016. Lidar guided stereo simultaneous localization and mapping (SLAM) for UAV outdoor 3-D scene reconstruction, Image and Vision Computing New Zealand (IVCNZ), pp.1-6. 